\title{
Determinants of Dirac Operators and Thirring Model Partition Functions on Riemann Surfaces with Boundaries
}

\author{
Siye $\mathrm{Wu}$ \\ Department of Mathematics, Massachusetts Institute of Technology, Cambridge, MA 02139, \\ USA
}

\begin{abstract}
The partition function of the Thirring model on a Riemann surface with boundaries is calculated using the method of Freedman and Pilch by introducing an auxiliary vector potential in the path integral of fermion representation. The Hodge decomposition on manifolds with boundaries is used to integrate over the harmonic forms. The result agrees with the bosonized calculation. The determinants of Dirac operators with mixed Neveu-Schwarz and Ramond boundary conditions are expressed in terms of the Riemann $\vartheta$-functions of the doubled surface.
\end{abstract}

\section{Introduction}

The determinants of the Laplacians and the Dirac operators on a closed Riemann surface have been studied extensively in the Polyakov formulation of the closed string theory $[1,2]$. For the open strings, the world sheets are Riemann surfaces with boundaries [3]. Determinants of the Laplacians with both Dirichlet and Neumann boundary conditions were calculated and used in the study of open bosonic strings $[4,5]$. In this paper, we calculate the determinants of Dirac operators with Neveu-Schwarz and Ramond boundary conditions. These determinants are useful in the open superstring theory, where the bosonic coordinates of the world sheet live on a Riemann surface with boundaries.

The determinants of Dirac operators coupled to an abelian gauge field on a closed Riemann surface with boundaries were calculated by Quillen's method of holomorphic anomaly $[6,2]$. The result was soon used to prove the bosonization of first order fermionic systems on a closed Riemann surface of higher genus [7,8]. Recently it was also used in the calculation of the partition function of the massless Thirring model in the fermion representation by introducing an auxiliary vector potential [9]. The same method works for the Thirring model on a Riemann surface with boundaries. We will calculate its partition using the fermion determinant and show that it agrees with the bosonized calculation. 
This paper is organized as follows. In Sect. 2, the geometry of Riemann surfaces with boundaries are studied by the doubling or the method of images [4]. In particular, the moduli of these surfaces can be described by the period matrices of the doubled surfaces. Hodge decompositions on manifolds with boundaries [10, 11] are applied to study harmonic 1 -forms on Riemann surfaces with boundaries. In Sect. 3, spin structures and boundary conditions on a Riemann surface with boundaries are discussed and the spinors with Neveu-Schwarz or Ramond boundary conditions are identified with the parity even sections of spinor bundles on the doubled surface. The determinant of the Dirac operator is obtained from the determinant on a closed Riemann surface $[2,9]$, since the Dirac operator on the doubled surface is diagonal under the parity decomposition. In Sect. 4, the partition function of the massless Thirring model on a Riemann surface with boundaries is computed using an auxiliary vector potential [9]. The fermion integral is the determinant calculated in Sect. 3. The Hodge decomposition with absolute boundary condition is used to integrate over the vector potential. Bosonization is treated in Sect. 5. The fermion determinant is replaced by the path integral of a circle-valued scalar coupled to the auxiliary potential [12]. Effectively, the theory is one of a free scalar which takes value in a circle of scaled radius. The partition function coincides with the fermion calculation after a Poisson resummation. In the Appendix, zeta-functions of the Laplacians with Dirichlet and Neumann boundary conditions at zero are calculated using the method of heat kernel expansion. These values are used in the main text.

\section{Geometry of Riemann Surfaces with Boundaries}

This section will introduce some machinery used in the calculation of the determinants of the Dirac operators and the partition function of the Thirring model.

Consider an oriented Riemann surface $M$ of genus $g$ but with $n+1$ boundaries $d_{k},(k=0,1, \ldots, n)$, i.e. $\partial M=\bigcup_{k=0}^{n} d_{k}$. Topologically, $M$ is characterized by two numbers, $g$ and $n$. More precisely, $M$ is topologically equivalent to the Riemann sphere with $g$ handles added but $n+1$ disks removed. Suppose the homology on the $g$ handles is generated by $a_{i}, b_{i}(i=1,2, \ldots, g)$. Then

$$
\operatorname{dim} H_{1}(M)=2 g+n,
$$

where $a_{i}, b_{i}(i=1, \ldots, n)$ and $d_{k}(k=1, \ldots, n)$ form a basis of $H_{1}(M)$. Note that $d_{0}$ is homologically dependent on $d_{1}, \ldots, d_{n}$.

Assume that $M$ satisfies the boundary condition for the world sheet of open string theory [3], i.e., the boundaries are geodesics on the surface or there is a tubular neighborhood such that the metric is a product [13]. By the Schwartz reflection principle, $M$ can be extended to a closed Riemann surface $\Sigma$, called the doubling of $M[4]$, with an anti-holomorphic involution $\sigma: \Sigma \rightarrow \Sigma, \sigma^{2}=1$ such that $M=\Sigma / \sigma$. The genus of $\Sigma$ is $2 g+n$. As is shown in Fig. 1, in addition to the generators in $H_{1}(M)$, the homology basis of $\Sigma$ consists of $a_{i}^{\prime}, b_{i}^{\prime}$, which are the mirror images of $a_{i}, b_{i}$ (with the orientation chosen to give the correct intersection 


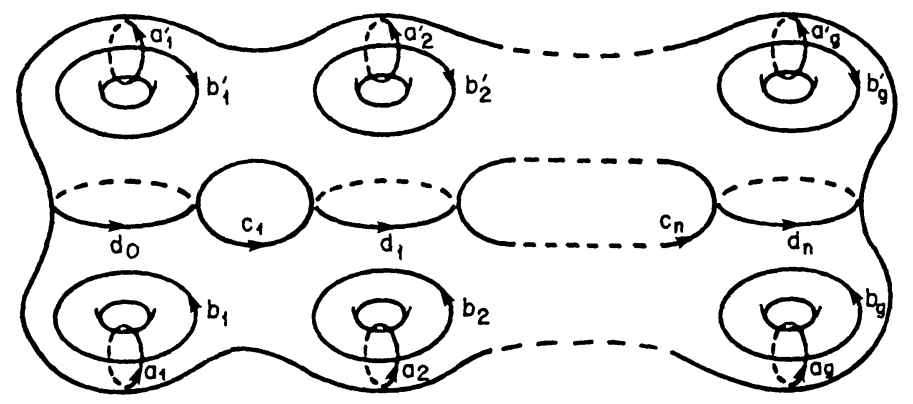

Fig. 1

numbers) and $c_{k},(k=1, \ldots, n)$ which intersect two boundaries. The map on $H_{1}(\Sigma)$ induced by $\sigma$ is given by

$$
\sigma_{*} a_{i}=-a_{i}^{\prime}, \sigma_{*} b_{i}=b_{i}^{\prime}, \sigma_{*} c_{k}=-c_{k}, \sigma_{*} d_{k}=d_{k},
$$

and $\sigma_{*}^{2}=1$

It is more convenient to study directly the geometry of the closed Riemann surface $\Sigma$ with an involution $\sigma$. Suppose $A_{I}, B_{I}(I=1, \ldots, 2 g+n)$ form an arbitrary homology basis of $\Sigma$ such that their intersection numbers are fixed by

$$
J\left(A_{I}, A_{J}\right)=J\left(B_{I}, B_{J}\right)=0, J\left(A_{I}, B_{J}\right)=-J\left(B_{I}, A_{J}\right)=\delta_{I J} .
$$

The induced map $\sigma_{*}$ is given by a matrix $\Gamma \in G L(2 g+n, \mathbb{Z})$,

$$
\sigma_{*} A_{I}=\Gamma_{I J} A_{J} .
$$

$\sigma^{2}=1$ implies $\Gamma^{2}=1$. Since $\sigma$ is orientation reversing, $\sigma_{*}$ changes the sign of the intersection numbers. Hence

$$
\sigma_{*} B_{I}=-\Gamma_{J I} B_{J}
$$

If $\Sigma$ is the doubling of $M$, we can choose

$$
A_{I}=\left\{\begin{array}{l}
a_{I}, I=1, \ldots, g \\
a_{I-g}^{\prime}, I=g+1, \ldots, 2 g \\
c_{I-2 g}, I=2 g+1, \ldots, 2 g+n
\end{array} \quad B_{I}=\left\{\begin{array}{l}
b_{I}, I=1, \ldots, g \\
b_{I-g}^{\prime}, I=g+1, \ldots, 2 g \\
d_{I-2 g}, I=2 g+1, \ldots, 2 g+n
\end{array}\right.\right.
$$

In this case

$$
\Gamma=\left(\begin{array}{ccc}
0 & -I_{g \times g} & 0 \\
-I_{g \times g} & 0 & 0 \\
0 & 0 & -I_{n \times n}
\end{array}\right)
$$

The period matrix of $\Sigma$ has some special properties because of the $\mathbb{Z}_{2}$-symmetry $[14,4]$. Suppose $\omega_{J}(J=1, \ldots, 2 g+n)$ form a basis of the holomorphic 1 -forms on $\Sigma$, normalized by $\int_{A_{I}} \omega_{J}=\delta_{I J}$. Then $\int_{B_{I}} \omega_{J}=\Omega_{I J}$ is the period matrix of $\Sigma$. Since $\sigma^{*} \bar{\omega}_{J}$ is also holomorphic, it can be expressed as a linear combination of $\omega_{J}$ 's. In fact

$$
\sigma^{*} \bar{\omega}_{J}=\Gamma_{I J} \omega_{I}
$$


This follows from a simple calculation

$$
\int_{A_{I}} \sigma^{*} \bar{\omega}_{J}=\int_{\sigma_{*} A_{I}} \bar{\omega}_{J}=\int_{\Gamma_{I K} A_{K}} \bar{\omega}_{J}=\Gamma_{I J}
$$

Furthermore,

$$
\bar{\Omega}_{I J}=\int_{B_{I}} \bar{\omega}_{J}=\int_{\sigma_{*} B_{I}} \sigma^{*} \bar{\omega}_{J}=-\Gamma_{K I} \Omega_{K L} \Gamma_{L J}
$$

or equivalently,

$$
\bar{\Omega}=-\Gamma^{T} \Omega \Gamma .
$$

If $\Sigma$ comes from doubling $M$ and $\Gamma$ is given by (2.7), then

$$
\Omega=\left(\begin{array}{ccc}
A & B & C \\
-\bar{B} & -\bar{A} & -\bar{C} \\
C^{T} & -\bar{C}^{T} & D
\end{array}\right),
$$

where $A=A^{T}, B=-\bar{B}^{T}$ are $g \times g$ matrices, $C$ is a $g \times n$ matrix and $D$ is an $n \times n$ matrix satisfying $\operatorname{Re} D=0$ and $D=D^{T}$.

Finally, we make a useful observation that $\operatorname{Im} \Omega$ is block diagonalized according to the projection operators $P_{ \pm}=\frac{1}{2}(1 \pm \Gamma)$, while $\operatorname{Re} \Omega$ is nonzero only in the off-diagonal blocks. To see this, write (2.9) in the following way:

$$
\operatorname{Re} \Omega-i \operatorname{Im} \Omega=-\left(P_{+}-P_{-}\right)(\operatorname{Re} \Omega+i \operatorname{Im} \Omega)\left(P_{+}-P_{-}\right) .
$$

A simple calculation yields

$$
\begin{aligned}
& \operatorname{Re} \Omega=P_{+} \operatorname{Re} \Omega P_{-}+P_{-} \operatorname{Re} \Omega P_{+}, P_{+} \operatorname{Re} \Omega P_{+}=0, P_{-} \operatorname{Re} \Omega P_{-}=0 \\
& \operatorname{Im} \Omega=P_{+} \operatorname{Im} \Omega P_{+}+P_{-} \operatorname{Im} \Omega P_{-}, P_{+} \operatorname{Im} \Omega P_{-}=0, P_{-} \operatorname{Im} \Omega P_{+}=0
\end{aligned}
$$

In particular,

$$
(\operatorname{Im} \Omega)^{-1}=P_{+}(\operatorname{Im} \Omega)^{-1} P_{+}+P_{-}(\operatorname{Im} \Omega)^{-1} P_{-} .
$$

The Hodge decomposition

$$
\Omega^{p}(M)=d \Omega^{p-1} \oplus \delta \Omega^{p+1} \oplus \operatorname{ker} \Delta^{p}, \operatorname{ker} \Delta^{p} \cong H^{p}(M)
$$

on a closed compact manifold $M$ is well known. To generalize it to the case of manifolds with boundaries, boundary conditions for $d$ or $\delta$ should be specified to make sure that $\delta$ is the adjoint of $d$ up to a minus sign. There are two ways to do this, corresponding to two different Hodge decompositions on manifolds with boundaries. We state the main results of the Hodge-de Rham theory for manifolds with boundaries but refer readers to the mathematical literature for proof $[10,11]$.

A differential $p$-form $\omega \in \Omega^{p}(M)$ on a Riemannian manifold with boundary $\partial M$ is called tangential at the boundary if any contraction with a vector field normal to the boundary vanishes. It is normal at $\partial M$ if it vanishes when contracted with any $p$ vector fields tangent to the boundary. A form $\omega \in \Omega^{p}(M)$ satisfies the relative boundary condition if both $\omega$ and $\delta \omega$ are normal at the boundary. It satisfies the absolute boundary condition if $\omega$ and $d \omega$ are tangential at the boundary. The spaces of forms with these boundary conditions are denoted by $\Omega_{\mathrm{rel}}^{p}(M)$ and $\Omega_{\mathrm{abs}}^{p}(M)$ respectively. 
Hodge Decomposition with Relative Boundary Condition. Let $M$ be a manifold with boundary $\partial M$. Suppose the operators $d_{\mathrm{rel}}: \Omega_{\mathrm{rel}}^{p-1}(M) \rightarrow \Omega^{p}(M)$ and $\Delta_{\mathrm{rel}}=d_{\mathrm{rel}} \delta$ $+\delta d_{\text {rel }}: \Omega_{\text {rel }}^{p}(M) \rightarrow \Omega^{p}(M)$ have relative boundary condition, then

$$
\Omega^{p}(M)=d_{\mathrm{rel}} \Omega_{\mathrm{rel}}^{p-1}(M) \oplus \delta \Omega^{p+1}(M) \oplus \operatorname{ker} \Delta_{\mathrm{rel}}^{p} .
$$

Furthermore, the space of harmonic forms with relative boundary condition is isomorphic to the relative cohomology group $H^{p}(M, \partial M)$.

Hodge Decomposition with Absolute Boundary Condition. Let $M$ be a manifold with boundary $\partial M$. Suppose the operators $\delta_{\mathrm{abs}}: \Omega_{\mathrm{abs}}^{p+1}(M) \rightarrow \Omega^{p}(M)$ and $\Delta_{\mathrm{abs}}=d \delta_{\mathrm{abs}}$ $+\delta_{\mathrm{abs}} d: \Omega_{\mathrm{abs}}^{p}(M) \rightarrow \Omega^{p}(M)$ have absolute boundary condition, then

$$
\Omega^{p}(M)=d \Omega^{p-1}(M) \oplus \delta_{\mathrm{abs}} \Omega_{\mathrm{abs}}^{p+1}(M) \oplus \operatorname{ker} \Delta_{\mathrm{abs}}^{p} .
$$

Furthermore, the space of harmonic forms with absolute boundary condition is isomorphic to the absolute cohomology group $H^{p}(M)$.

For a Riemann surface $M$ of genus $g$ with $n+1$ boundaries,

$$
\operatorname{dim} H^{1}(M, \partial M)=\operatorname{dim} H^{1}(M)=2 g+n .
$$

So the number of harmonic 1 -forms with relative and absolute boundary conditions are both $2 g+n$. Since the absolute boundary condition appears naturally in the sections below, we will study it in more detail. A 1-form $A$ can be written as

$$
A=d s+* d p+h
$$

where $s \in \Omega^{0}(M)$ is a function, $h \in \operatorname{ker} \Delta_{\mathrm{abs}}^{1}$ is harmonic with absolute boundary condition and $* p \in \Omega_{\mathrm{abs}}^{2}(M)$ or $p \in \Omega_{D}^{0}(M)$ is a function satisfying the Dirichlet boundary condition $\left.p\right|_{\partial M}=0$.

To construct an explicit basis for the harmonic forms in $\operatorname{ker} \Delta_{\mathrm{abs}}^{1}$, we consider the doubled surface $\Sigma$ of $M$. Since a harmonic form $h$ on $\Sigma$ with the property $\sigma^{*} h=h$ satisfies the absolute boundary condition when restricted to $M$, it suffices to look for harmonic forms invariant under the involution. There are $4 g+2 n$ harmonic 1 -forms $\alpha_{i}, \alpha_{i}^{\prime}, \beta_{i}, \beta_{i}^{\prime}, \gamma_{k}, \delta_{k}(i=1, \ldots, g, k=1, \ldots, n)$ on $\Sigma$, dual to the homology basis shown in Fig. 1 , such that

$$
\int_{a_{\imath}} \alpha_{j}=\int_{a_{i}^{\prime}} \alpha_{j}^{\prime}=\int_{b_{i}} \beta_{j}=\int_{b_{i}^{\prime}} \beta_{j}^{\prime}=\delta_{i j}, \int_{c_{k}} \gamma_{l}=\int_{d_{k}} \delta_{l}=\delta_{k l}, \text { others }=0
$$

The $2 g+n$ linear combinations

$$
\tilde{\alpha}_{i}=\alpha_{i}-\alpha_{i}^{\prime}, \widetilde{\beta}_{i}=\beta_{i}+\beta_{i}^{\prime}, \widetilde{\delta}_{k}=\sqrt{2} \delta_{k}
$$

are invariant under the involution $\sigma^{*}$, hence they form a basis of the harmonic forms with absolute boundary condition on $M$. Every harmonic form $h \in \operatorname{ker} \Delta_{\text {abs }}^{1}$ can be written as

$$
\begin{aligned}
h & =2 \pi\left(\sum_{i=1}^{g} u_{i} \tilde{\alpha}_{i}-\sum_{i=1}^{g} v_{i} \widetilde{\beta}_{i}-\sum_{k=1}^{n} w_{k} \tilde{\delta}_{k}\right) \\
& =2 \pi\left[\left(\alpha, \alpha^{\prime}, \gamma\right) \cdot U-\left(\beta, \beta^{\prime}, \delta\right) \cdot V\right],
\end{aligned}
$$


where

satisfy

$$
U=\left(\begin{array}{c}
u \\
-u \\
0
\end{array}\right), \quad V=\left(\begin{array}{c}
v \\
v \\
\sqrt{2 w}
\end{array}\right)
$$

$$
P_{+} U=U, P_{-} U=0, P_{-} V=V, P_{+} V=0 .
$$

The norm of $h$ on $M$ is half of its norm on $\Sigma$ given by [9]

$$
\langle h, h\rangle_{\Sigma}=4 \pi^{2}(U \bar{\Omega}+V)(\operatorname{Im} \Omega)^{-1}(\Omega U+V) .
$$

A simple calculation shows

$$
\langle h, h\rangle_{M}=2 \pi^{2}\left[U P_{+} \operatorname{Im} \Omega P_{+} U+(U \Omega+V) P_{-}(\operatorname{Im} \Omega)^{-1} P_{-}(\Omega U+V)\right] .
$$

\section{Spin Structures and Determinants}

\section{of Dirac Operators on Riemann Surfaces with Boundaries}

In this section, we will study the spin structures on a Riemann surface with boundaries and calculate the determinant of the Dirac operator with mixed Ramond and Neveu-Schwarz boundary conditions.

It is well known that the number of inequivalent spin structures on a compact Riemannian manifold is equal to the number of the elements of the group $H^{1}\left(M, \mathbb{Z}_{2}\right)$ when the second Stiefel-Whitney class $w_{2}(M) \in H^{2}\left(M, \mathbb{Z}_{2}\right)$ vanishes. Intuitively, this is because there are two inequivalent choices of sign along each homologically nontrivial loop. However, there is not a canonical spinor bundle, except when the manifold is flat, like a torus. More precisely, $H^{1}\left(M, \mathbb{Z}_{2}\right)$ is in 1-1 correspondence with the set of the differences of the spinor bundles.

Fortunately, for a closed Riemann surface of genus $h$, there is a more definite way to describe the $2^{2 h}$ spinor bundles, provided that a homology basis is chosen. To see this, recall the Jacobian map $I$ from the set of divisors of degree zero to the Jacobi variety $J(\Sigma)=\mathbb{C}^{h} / \mathbb{Z}^{h}+\Omega \mathbb{Z}^{h}$ defined by

$$
I(D)=\int_{c} \omega \in J(\Sigma),
$$

where $c$ is a 1-cycle such that $\partial c=D$. It is a miracle of two-dimension that the chiral decomposition of the spinor bundle $S=S_{+} \oplus S_{-}$is also a decomposition to the holomorphic and anti-holomorphic line bundles [2]. Denote $D_{\alpha}$ the divisor of the holomorphic piece, where $\alpha$ labels spin structures. There is a 1-1 correspondence between $D_{\alpha}$ and a symmetric translate $[15,2,8]$

$$
e_{\alpha}=\Delta-I\left[D_{\alpha}-(h-1) P_{0}\right]
$$

of the $\Theta$-divisor [16], a complex variety in $J(\Sigma)$ of codimension one which consists of the zeros of the Riemann $\vartheta$-function. Here $P_{0} \in \Sigma$ is an arbitrary fixed point and $\Delta$ is the vector of Riemann constants in the Riemann vanishing theorem $[17,14]$. Hence a spin structure $D_{\alpha}$ can be described by two characteristics $E, F \in\left(\frac{1}{2} \mathbb{Z} / \mathbb{Z}\right)^{h}$ 


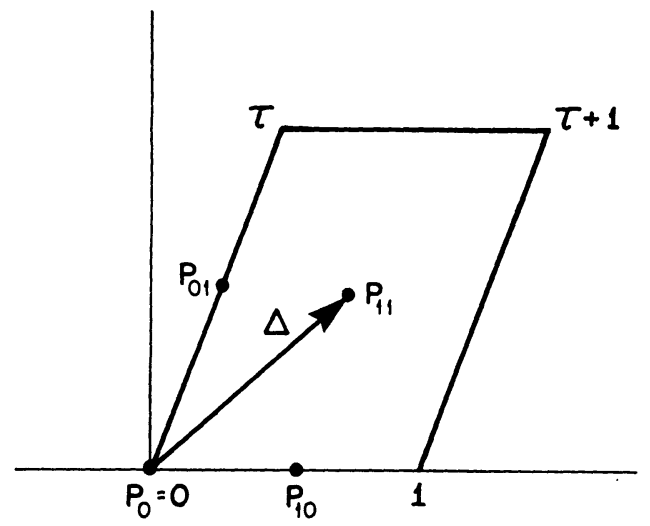

Fig. 2

defined by $e_{\alpha}=\Omega E+F$. In particular, there is a preferred spin structure $D_{0}$ which corresponds to $E=F=0$.

If $\Sigma$ is a torus with moduli parameter $\tau, J(\Sigma)$ is holomorphic equivalent to $\Sigma$. Choose $P_{0}=0, P_{11}=\frac{1}{2}+\frac{1}{2} \tau, P_{10}=\frac{1}{2}, P_{10}=\frac{1}{2} \tau$ as shown in Fig. 2 . The vector of Riemann constants is $\Delta=\vec{P}_{0} P_{11}$. The preferred spin structure is $D_{0}=\frac{1}{2} P_{11}-\frac{1}{2} P_{0}$, the spinor bundle of anti-periodic conditions along both homology loops. Nonzero symmetric translates of the $\Theta$-divisor $\Theta=\left\{P_{11}\right\}$ correspond to other spin structures $\frac{1}{2} P_{10}-\frac{1}{2} P_{0}, \frac{1}{2} P_{01}-\frac{1}{2} P_{0}, 0$.

To describe boundary conditions of spinors, choose an orthonormal frame $\left\{e_{a}, a=1,2\right\}$ near the boundary such that on $\partial M, e_{1}$ is tangent to the boundary and $e_{2}$ is the outer normal direction. Choose a spinor basis such that the representation of the Clifford algebra is given by two Pauli matrices $\sigma_{1}, \sigma_{2}$. Then the chiral operator $\gamma_{5}=\sigma_{3}$ is diagonal. If the spinor $\psi=\left(\begin{array}{c}\psi_{+} \\ \psi_{-}\end{array}\right)$satisfies $\psi_{+}=\psi_{-}$or $\psi_{+}=-\psi_{-}$on the boundary, we say it satisfies the Ramond or Neveu-Schwarz boundary condition respectively. Since the relative phase of $\psi_{+}$and $\psi_{-}$is not yet fixed, we can always require $\psi$ to be Ramond on one of the connected components of $\partial M$, say $d_{0}$. These boundary conditions are necessary to make sure that the boundary terms from the variation of the action vanish to get the correct equations of motion [18]. Mathematically, it turns out that the Dirac operators with these boundary conditions are self-adjoint.

Next, we define a spinor bundle $\mathscr{S}$ on the doubled surface $\Sigma=M \cup M^{\prime}$, where $M^{\prime}=\sigma(M)$, using the spin structure and the boundary condition on $M$. The map $\sigma: M \rightarrow M^{\prime}$ defines a pull-back bundle $S^{\prime}=\sigma^{*} S$ on $M^{\prime}$. In fact $\sigma^{*} S_{ \pm}^{\prime}=S_{\mp}$, since $\sigma$ is orientation reversing. If the spinor bundle $\mathscr{S}$ on $\Sigma$ satisfies $\left.\mathscr{S}\right|_{M}=S$ and $\left.\mathscr{S}\right|_{M^{\prime}}=S^{\prime}$, then its characteristics must have the form

$$
E=\left(\begin{array}{c}
\varepsilon \\
-\varepsilon \\
\delta
\end{array}\right), \quad F=\left(\begin{array}{l}
\kappa \\
\kappa \\
\lambda
\end{array}\right),
$$


where $\varepsilon, \kappa \in\left(\frac{1}{2} \mathbb{Z} / \mathbb{Z}\right)^{g}, \lambda \in\left(\frac{1}{2} \mathbb{Z} / \mathbb{Z}\right)^{n}$ describe the spin structure of $S$ over $M$, while $\delta \in\left(\frac{1}{2} \mathbb{Z} / \mathbb{Z}\right)^{n}$ describes the spin structure along the new loops $c_{k}$, which depends on how the two spin bundles $S$ and $S^{\prime}$ are glued together on the boundary $\partial M$.

The glueing is done according to the type of boundary conditions. If the spinor $\psi$ is Ramond on $d_{k}$, we identify $\psi=\left(\begin{array}{c}\psi_{+} \\ \psi_{-}\end{array}\right) \in S$ and $\psi^{\prime}=\left(\begin{array}{c}\psi_{+}^{\prime} \\ \psi_{-}^{\prime}\end{array}\right) \in S^{\prime}$ on $d_{k}$ iff $\psi_{ \pm}=\psi_{ \pm}^{\prime}$. If $\psi$ is Neveu-Schwarz on $d_{k}$, we identify them iff $\psi_{ \pm}=-\psi_{ \pm}^{\prime}$. Thus the spin structure along $c_{k}$ depends on the type of boundary conditions on $\partial M$. For example, if $M$ is a cylinder with two boundaries $d_{0}$ and $d_{1}$, then $\Sigma$ is a torus with homology basis $c_{1}, d_{1}$. Ramond boundary condition is required on $d_{0}$. If the spinor is Neveu-Schwarz on $d_{1}$, then it is anti-periodic along $c_{1}$, hence $\delta=0$. On the other hand, if the spinor is Ramond on $d_{1}$, then $\delta=\frac{1}{2}$. In general, if $\partial M$ has $n+1$ connected components $d_{k}, k=1, \ldots, n$, then $\delta_{k}=0$ or $\frac{1}{2}$ depending on whether the boundary conditions on $d_{k-1}$ and $d_{k}$ are of the same type or not.

The motivation for the above construction of $\mathscr{S}$ is that we can identify spinors on $M$ satisfying the desired boundary conditions with the spinors on $\Sigma$ which are even under parity. The parity of the spinors makes sense because the pull-back bundle $\sigma^{*} \mathscr{S}$ is isomorphic to $\mathscr{S}$, as we show below. Consider a holomorphic line bundle $L$ with a divisor $D$ of degree $r$ over a Riemann surface $\Sigma$ with an involution $\sigma$. Then $\sigma^{*} \bar{L}$ is also holomorphic, with divisor $\sigma^{*} \bar{D}$. Here bar means complex conjugation. The images under the Jacobian map are related by

$$
\left.I\left(\sigma^{*} \bar{D}-r \sigma\left(P_{0}\right)\right)=\int_{\sigma_{*} \bar{c}} \omega=\overline{\int_{c} \sigma^{*} \bar{\omega}}=\overline{\int_{c} \Gamma^{T} \omega}=\Gamma^{T} \overline{I\left(D-r P_{0}\right.}\right) .
$$

If $\mathscr{S}$ is a spinor bundle of characteristics $E, F$ given by (3.3), then the symmetric translate of $\Theta$ corresponding to $\sigma^{*} \mathscr{S}$ is

$$
\Delta+\Gamma^{T}(\overline{\Omega E+F})-\Gamma^{T} \bar{\Delta}=\Delta-\Gamma^{T} \bar{\Delta}-(\Omega \widetilde{E}+F),
$$

where

$$
\tilde{E}=\left(\begin{array}{c}
\varepsilon \\
-\varepsilon \\
-\delta
\end{array}\right) .
$$

This is the same as $\Omega E+F$ modulo the period lattice $L_{\Omega}=\mathbb{Z}^{4 g+2 n}+\Omega \mathbb{Z}^{4 g+2 n}$, because

$$
\Gamma^{T} \bar{\Delta} \equiv \Delta\left(\bmod L_{\Omega}\right)
$$

which follows from

$$
\overline{\vartheta(z \mid \Omega)}=\vartheta\left(\Gamma^{T} \bar{z} \mid \Omega\right)
$$

and the Riemann vanishing theorem. This proves $\sigma^{*} \mathscr{S} \cong \mathscr{S}$, hence $\sigma^{*}$ maps sections of $\mathscr{S}$ to themselves. Since $\left(\sigma^{*}\right)^{2}=1$, the bundle decomposes into the parity even and odd parts, $\mathscr{S}=\mathscr{S}^{\mathrm{ev}} \oplus \mathscr{S}^{\text {od }}$, where

$$
\mathscr{S}^{\mathrm{ev}}=\left\{\psi \in \mathscr{S} \mid \sigma^{*} \psi=\psi\right\}, \mathscr{S}^{\mathrm{od}}=\left\{\psi \in \mathscr{S} \mid \sigma^{*} \psi=-\psi\right\} .
$$

This decomposition is different from the chiral decomposition. 
The local frame $\left\{e_{a}\right\}$ on $M$ near $\partial M$ can be extended continuously to a frame defined on a tubular neighborhood $\partial M \times(-\varepsilon, \varepsilon)$ of $\partial M$ on $\Sigma, e_{2}$ being the inner normal direction of $M^{\prime}$ on $\partial M$. Locally, the parity operator $\sigma^{*}$ is given by

$$
\sigma^{*} \psi(p, y)= \pm \sigma_{1} \psi(p,-y), p \in d_{k}, y \in(-\varepsilon, \varepsilon) .
$$

The \pm sign is chosen depending on whether there is a twist when the spinors are glued on $d_{k}$, or equivalently whether the spinor is Ramond or Neveu-Schwarz on $d_{k}$. In both cases, the boundary condition is equivalent to

$$
\sigma^{*} \psi(p, 0)=\psi(p, 0), p \in d_{k} .
$$

Hence the spinor on $M$ with the desired boundary condition is equivalent to a parity even spinor on $\Sigma$, i.e. a section of $\mathscr{S}^{\text {ev }}$.

Finally, we study the Dirac operator on a Riemann surface with boundaries. Recall that the determinant of the non-chiral Dirac operator coupled to a $U(1)$ gauge field on a closed Riemann surface $\Sigma$ is given by $[2,9,19]$

$$
\operatorname{det} \Phi_{\Sigma}(A)=\exp \left[-\frac{1}{2 \pi} \int_{\Sigma} d p \wedge * d p\right]\left(\frac{\operatorname{det}(\operatorname{Im} \Omega) \operatorname{vol}(\Sigma)}{\operatorname{det}^{\prime} \Delta_{\Sigma}}\right)^{1 / 2}\left|\vartheta\left[\begin{array}{l}
U+E \\
V+F
\end{array}\right](0 \mid \Omega)\right|^{2},
$$

where $\Omega$ is the period matrix of $\Sigma, E, F$ are characteristics of the spin structure and $p, U, V$ appear in the Hodge decomposition of $A$

$$
A=d s+* d p+2 \pi(\alpha \cdot U-\beta \cdot V) .
$$

To calculate $\operatorname{det} \mathbb{D}_{M}(A)$ with the required boundary condition, we should study more closely the boundary condition and choose the appropriate type of Hodge decomposition for the potential $A$. Since $p$ is the infinitesimal generator of a local chiral transformation, which changes the relative phase of $\psi_{+}$and $\psi_{-}$, the boundary condition on spinors requires $\left.p\right|_{\partial M}=0$. Thus we shall use the Hodge decomposition with absolute boundary condition. On the other hand, $s$ is the infinitesimal generator of a local gauge transformation, which only changes the overall phase of $\psi$. We will make a technical assumption to be justified later, that the 1-form $A$ satisfies the absolute boundary condition on $\partial M$, or equivalently, the function $s$ satisfies the Neumann boundary condition $\left.\frac{\partial s}{\partial n}\right|_{\partial M}=0$. In this case, $A$ can be extended to a smooth 1-form on $\Sigma$ satisfying $\sigma^{*} A=A$. Moreover, the Dirac operator $\mathbb{D}_{\Sigma}(A)$ commutes with the parity operator $\sigma^{*}$, hence it is diagonalized under the parity decomposition $\mathscr{S}=\mathscr{S}^{\mathrm{ev}} \oplus \mathscr{S}^{\mathrm{od}}$,

$$
\mathbb{D}_{\Sigma}(A)=\not^{\mathrm{ev}} \oplus \mathbb{D}^{\mathrm{od}},
$$

where

$$
\not^{\mathrm{ev}}: \mathscr{S}^{\mathrm{ev}} \rightarrow \mathscr{S}^{\mathrm{ev}}, \not^{\mathrm{od}}: \mathscr{S}^{\mathrm{od}} \rightarrow \mathscr{S}^{\mathrm{od}} .
$$

Both $\not D^{\text {ev }}$ and $\not \not^{\text {od }}$ are self-adjoint operators and have well-posed eigenvalue problems. Hence

$$
\operatorname{det} \not_{\Sigma}(A)=\operatorname{det} \not{ }^{\text {ev }} \cdot \operatorname{det} \not D^{\text {od }} .
$$

The chiral operator $\gamma_{5}$ anti-commutes with both the parity $\sigma^{*}$ and the Dirac operator $\not_{\Sigma}(A)$. This implies that $\gamma_{5}: \mathscr{S}^{\mathrm{ev}} \rightarrow \mathscr{S}^{\text {od }}$ is a bundle isomorphism and that 
the spectrum of $\not^{\text {ev }}$ is exactly the negative of that of $\not^{\text {od }}$. Up to a possible sign, we have

$$
\operatorname{det} \not^{\mathrm{ev}}=\operatorname{det} \not D^{\mathrm{od}}
$$

Hence

$$
\operatorname{det} \not^{\mathrm{ev}}=\left(\operatorname{det} \not_{\Sigma}(A)\right)^{1 / 2} .
$$

Notice that the choice of signs in (3.17) and (3.18) is only a matter of convention; this is different from the sign ambiguity in Witten's $S U(2)$ anomaly [20] due to non-trivial global gauge transformations.

If $\Omega$ is the period matrix of the Riemann surface with involution, $U, V$ come from a harmonic form with absolute boundary condition and $E, F$ are characteristics of a parity invariant spinor bundle, then the Riemann $\vartheta$-function in (3.12) is real. In fact,

$$
\begin{aligned}
\overline{\vartheta\left[\begin{array}{l}
U+E \\
V+F
\end{array}\right](0 \mid \Omega)} & =\overline{\vartheta(\Omega(U+E)+V+F \mid \Omega)}=\vartheta(\Omega(U+\tilde{E})+V+F \mid \Omega) \\
& =\vartheta\left[\begin{array}{l}
U+\tilde{E} \\
V+F
\end{array}\right](0 \mid \Omega)=\vartheta\left[\begin{array}{l}
U+E \\
V+F
\end{array}\right](0 \mid \Omega) .
\end{aligned}
$$

Since the spinors on $M$ with the required boundary condition are identified with the parity even spinors on $\Sigma, \operatorname{det} \not_{M}(A)=\operatorname{det} \not^{\text {ev }}$, our final result is

$$
\operatorname{det} D_{M}(A)=\exp \left[-\frac{1}{2 \pi} \int_{M} d p \wedge * d p\right]\left(\frac{2 \operatorname{det}(\operatorname{Im} \Omega) \operatorname{vol}(M)}{\operatorname{det}^{\prime} \Delta_{\Sigma}}\right)^{1 / 4} \vartheta\left[\begin{array}{l}
U+E \\
V+F
\end{array}\right](0 \mid \Omega)
$$

The modular invariance of $\operatorname{det} \Phi_{M}(A)$ follows from that of $\operatorname{det} D_{\Sigma}(A)$. It is also easy to see directly. Under a modular transformation $\left(\begin{array}{ll}D & C \\ B & A\end{array}\right) \in S p(4 g+2 n, \mathbb{Z})$, the
$\vartheta$-function transforms as $[21,2]$

$$
\vartheta\left[\begin{array}{l}
U^{\prime}+E^{\prime} \\
V^{\prime}+F^{\prime}
\end{array}\right]\left(0 \mid \Omega^{\prime}\right)=(\text { phase }) \operatorname{det}(C \Omega+D)^{1 / 2} \vartheta\left[\begin{array}{c}
U+E \\
V+F
\end{array}\right](0 \mid \Omega),
$$

where $U^{\prime}, V^{\prime}$ given by

$$
\left(\begin{array}{l}
U^{\prime} \\
V^{\prime}
\end{array}\right)=\left(\begin{array}{cc}
D & -C \\
-B & A
\end{array}\right)\left(\begin{array}{l}
U \\
V
\end{array}\right)
$$

are the coefficients of the harmonic form under the new basis and $E^{\prime}, F^{\prime} \in\left(\frac{1}{2} \mathbb{Z}\right)^{2 g+n}$ depend only on $E, F$ and the modular transformation. The factor after the phase is canceled by the contribution from the change in $\operatorname{det}(\operatorname{Im} \Omega)^{1 / 4}$. Finally, $U^{\prime}, V^{\prime}$ satisfy the same property (2.23) as $U, V$ if the modular transformation commutes with $\sigma_{*}$ on the homology basis. In this case, the phase and the determinant in (3.21) are separately real.

\section{Calculation of the Thirring Model Partition Functions}

The determinants of Dirac operators on closed Riemann surfaces were used in calculating the partition functions of the massless Thirring model in the fermion representation [9]. An auxiliary vector potential was introduced in the fermionic 
path integral. The functional integral over the fermion field is the determinant of the Dirac operator coupled to the gauge potential. The integral over the auxiliary field was calculated using the Hodge decomposition, integrating separately over the exact, coexact as well as the harmonic components. In an earlier work [22], a similar method was used to calculate the partition function of fermions coupled to gauge fields in superstring theory. However, a sum over integer and half-integer harmonic components is used and the partition function obtained there was rather different from [9].

In this section, we compute the partition of the Thirring model on a Riemann surface with boundaries. Consider the following path integral

$$
Z(Q)=\frac{\int \mathscr{D} A_{\alpha} \mathscr{D} \bar{\psi} \mathscr{D} \psi \mathrm{e}^{-\int_{M} d^{2} \sigma \sqrt{g}\left[\frac{1}{2 \pi Q} g^{\alpha \beta} A_{\alpha} A_{\beta}-\bar{\psi} \gamma^{\alpha}\left(D_{\alpha}+i A_{\alpha}\right) \psi\right]}}{\int \mathscr{D} A_{\alpha} \mathrm{e}^{-\int_{M} d^{2} \sigma \sqrt{g} \frac{1}{2 \pi Q} g^{\alpha \beta} A_{\alpha} A_{\beta}}},
$$

where $g_{\alpha \beta}$ is the metric on $M$ and $A_{\alpha}$ is an auxiliary vector potential. The partition function of the Thirring model with coupling constant $Q$

$$
Z(Q)=\int \mathscr{D} \bar{\psi} \mathscr{D} \psi \mathrm{e}^{-\int_{M}^{d^{2} \sigma \sqrt{g}\left[\bar{\psi} \gamma^{\alpha} D_{\alpha} \psi-\frac{1}{2} \pi Q \bar{\psi} \gamma^{\alpha} \psi \bar{\psi} \gamma_{\alpha} \psi\right]}}
$$

can be recovered if one performs the integral over $A_{\alpha}$ after a Gaussian shift $A_{\alpha} \rightarrow A_{\alpha}$ $-i \pi Q \bar{\psi} \gamma_{\alpha} \psi$. Because of the Neveu-Schwarz or Ramond boundary condition on the fermion $\psi$, the shifting $-i \pi Q \bar{\psi} \gamma_{\alpha} \psi d \sigma^{\alpha}$ is a 1-form satisfying the absolute boundary condition. Hence we only have to integrate over such $A$ in (4.1), which justifies an assumption we made before.

Changing the order of the integrations, the integration over the fermion $\psi$ is

$$
\int \mathscr{D} \bar{\psi} \mathscr{D} \psi \mathrm{e}^{\int_{M} d^{2} \sigma \sqrt{g} \bar{\psi} \gamma^{\alpha}\left(D_{\alpha}+i A_{\alpha}\right) \psi}=\operatorname{det} \not_{M}(A),
$$

the determinant of the Dirac operator (3.20) calculated in Sect. 3. The integral over $A_{\alpha}$ can be transformed, via Hodge decomposition (2.18), into the integrations over the non-zero modes of $s \in \Omega_{N}^{0}(M), p \in \Omega_{D}^{0}(M)$ and the harmonic forms $h \in \operatorname{ker} \Delta_{\mathrm{abs}}^{1}$, or equivalently, over $u, v, w$ in (2.21). The Jacobian involved in this transformation is independent of the integration variables and therefore cancels between the numerator and the denominator in (4.1). Thus

$$
\begin{aligned}
& Z(Q)=\frac{\int \mathscr{D} A_{\alpha} \mathrm{e}^{-\int_{M} d^{2} \sigma \sqrt{g} \frac{1}{2 \pi Q} g^{\alpha \beta} A_{\alpha} A_{\beta}} \operatorname{det} D_{A}(M)}{\int \mathscr{D} A_{\alpha} \mathrm{e}^{-\int_{M} d^{2} \sigma \bar{g} \frac{1}{2 \pi Q} g^{\alpha \beta} A_{\alpha} A_{\beta}}} \\
&=\frac{\int \mathscr{D} S \mathscr{D} p d u d v d w \exp \left\{-\frac{1}{2 \pi Q}\left[\langle d s, d s\rangle_{M}+\langle d p, d p\rangle_{M}+2 \pi^{2}(U \bar{\Omega}+V)(\operatorname{Im} \Omega)^{-1}(\Omega U+V)\right]\right\}}{\int \mathscr{D} S \mathscr{D} p d u d v d w \exp \left\{-\frac{1}{2 \pi Q}\left[\langle d s, d s\rangle_{M}+\langle d p, d p\rangle_{M}+2 \pi^{2}(U \bar{\Omega}+V)(\operatorname{Im} \Omega)^{-1}(\Omega U+V)\right]\right\}} \\
& \quad \times\left(\frac{2 \operatorname{det}(\operatorname{Im} \Omega) \operatorname{vol}(M)}{\operatorname{det} \Delta_{\Sigma}}\right)^{1 / 4} \exp \left[-\frac{1}{2 \pi}\langle d p, d p\rangle_{M}\right] \vartheta\left[\begin{array}{l}
U+E \\
V+F
\end{array}\right](0 \mid \Omega) \\
&=\left(\frac{2 \operatorname{det}(\operatorname{Im} \Omega) \operatorname{vol}(M)}{\operatorname{det}^{\prime} \Delta_{\Sigma}}\right)^{1 / 4}\left(\frac{\operatorname{det} \frac{1}{Q} \Delta_{D}}{\operatorname{det}\left(\frac{1}{Q}+1\right) \Delta_{D}}\right)^{1 / 2} \sum_{N \in \mathbb{Z}^{2} g+n} \frac{\mathcal{N}_{N}}{\mathscr{D}},
\end{aligned}
$$


where

$$
\begin{aligned}
\mathscr{N}_{N}= & \int d u d v d w \exp \left[-\frac{\pi}{Q}(U \bar{\Omega}+V)(\operatorname{Im} \Omega)^{-1}(\Omega U+V)\right. \\
& +i \pi(N+U+E) \Omega(N+U+E)+2 \pi i(N+U+E)(V+F)]
\end{aligned}
$$

and

$$
\mathscr{D}=\int d u d v d w \exp \left[-\frac{\pi}{Q}(U \bar{\Omega}+V)(\operatorname{Im} \Omega)^{-1}(\Omega U+V)\right] .
$$

The determinant of the Laplacian with Dirichlet boundary condition was calculated in $[4,5]$, but we only need the following property from the zeta-function regularization [23].

$$
\operatorname{det}^{\prime}(c A)=c^{\zeta_{A}(0)} \operatorname{det}^{\prime} A,
$$

where $\zeta_{A}(s)$ is the zeta-function of a self-adjoint operator $A$. Therefore,

$$
Z(Q)=\left(\frac{2 \operatorname{det}(\operatorname{Im} \Omega) \operatorname{vol}(M)}{\operatorname{det}^{\prime} \Delta_{\Sigma}}\right)^{1 / 4}(1+Q)^{-\frac{1}{2} \zeta_{\Delta_{D}}(0)} \sum_{N} \frac{\mathscr{N}_{N}}{\mathscr{D}}
$$

The value $\zeta_{\Delta_{D}}(0)=-\frac{1}{6}(2 g+n-1)$ is derived in the Appendix. A straightforward calculation shows

$$
\begin{aligned}
& \mathscr{N}_{N}= \int d u d v d w \exp \left\{-\pi\left(\frac{1}{Q}+1\right)\left[U+\frac{1}{\frac{1}{Q}+1}(N+E)\right] P_{+} \operatorname{Im} \Omega P_{+}\left[U+\frac{1}{\frac{1}{Q}+1}(N+E)\right] .\right. \\
&\left.-\frac{\pi}{Q}\left[U \Omega+V-i Q(N+E) P_{-} \operatorname{Im} \Omega\right] P_{-}(\operatorname{Im} \Omega)^{-1} P_{-}\left[\Omega U+V-i Q \operatorname{Im} \Omega P_{-}(N+E)\right]\right\} \\
& \times \exp \left[-\frac{\pi}{1+Q}(N+E) P_{+} \operatorname{Im} \Omega P_{+}(N+E)-\pi(1+Q)(N+E) P_{-} \operatorname{Im} \Omega P_{-}(N+E)\right. \\
&\left.+2 \pi i(N+E) P_{+} \operatorname{Re} \Omega P_{-}(N+E)+2 \pi i(N+E) F\right], \\
& \mathscr{D}=\int d u d v d w \exp \left\{-\frac{\pi}{Q}\left[U P_{+} \operatorname{Im} \Omega P_{+} U+(U \Omega+V) P_{-}(\operatorname{Im} \Omega)^{-1} P_{-}(\Omega U+V)\right]\right\} .
\end{aligned}
$$

The finite dimensional Gaussian integrals on $u$ and $v, w$ over the subspaces im $P_{+}$ and $\operatorname{im} P_{-}$cancel between the numerator and the denominator up to some scaling factors. Thus

$$
\begin{aligned}
Z(Q)= & \left(\frac{2 \operatorname{det}(\operatorname{Im} \Omega) \operatorname{vol}(M)}{\operatorname{det}^{\prime} \Delta_{\Sigma}}\right)^{1 / 4}(1+Q)^{-\frac{1}{2} \zeta_{\Delta_{D}}(0)}\left(\frac{\frac{1}{Q}}{\frac{1}{Q}+1}\right)^{g / 2} \\
& \times \sum_{N} \exp [i \pi(N+E) \tilde{\Omega}(N+E)+2 \pi i(N+E) F] \\
= & \left(\frac{2 \operatorname{det}(\operatorname{Im} \Omega) \operatorname{vol}(M)}{\operatorname{det}^{\prime} \Delta_{\Sigma}}\right)^{1 / 4}(1+Q)^{-\frac{1}{2} \zeta_{\Delta_{D}}(0)-\frac{g}{2}} \vartheta\left[\begin{array}{l}
E \\
F
\end{array}\right](0 \mid \tilde{\Omega}),
\end{aligned}
$$


where

$$
\widetilde{\Omega}=\operatorname{Re} \Omega+\frac{i}{1+Q} P_{+} \operatorname{Im} \Omega P_{+}+i(1+Q) P_{-} \operatorname{Im} \Omega P_{-}
$$

is the period matrix $\Omega$ rescaled differently on the two subspaces $\operatorname{im} P_{+}$. When $Q>-1, \widetilde{\Omega}$ remains in the Siegel upper half space, hence the infinite sum over $N$ converges. This agrees with the observation in [9].

The modular invariance of the partition function can been seen from (4.4). Since the norm of the harmonic form is not changed under modular transformation and the Jacobian from changing the variables $u, v, w$ cancels between the numerator and the denominator in (4.4), the same argument for the modular invariance of $\operatorname{det} D_{M}(A)$ applies here.

As an interesting check of the main result (4.11), we consider two special cases. First, if $M$ is a closed Riemann surface of genus $g$, then $\Sigma$ is the disconnected union of $M$ and its conjugate. There are no relations between the harmonic forms on one connected component and those on the other. Thus the period matrix of $\Sigma$ is

$$
\Omega=\left(\begin{array}{cc}
\Omega_{M} & 0 \\
0 & -\bar{\Omega}_{M}
\end{array}\right)
$$

where $\Omega_{M}$ is the period matrix of $M$. Furthermore,

$$
E=\left(\begin{array}{c}
\varepsilon \\
-\varepsilon
\end{array}\right), F=\left(\begin{array}{l}
\kappa \\
\kappa
\end{array}\right), P_{ \pm}=\frac{1}{2}\left(\begin{array}{cc}
I & \mp I \\
\mp I & I
\end{array}\right), N=\left(\begin{array}{c}
m \\
-n
\end{array}\right),
$$

where $m, n \in \mathbb{Z}^{g}$. Using

$$
\operatorname{det}(\operatorname{Im} \Omega)=\left(\operatorname{det}\left(\operatorname{Im} \Omega_{M}\right)\right)^{2} \operatorname{det}^{\prime} \Delta_{\Sigma}=\left(\operatorname{det}^{\prime} \Delta_{M}\right)^{2},
$$

one can check (4.11) reproduces

$$
\begin{aligned}
Z(Q)= & 2^{1 / 4}\left(\frac{\operatorname{det}\left(\operatorname{Im} \Omega_{M}\right) \operatorname{vol}(M)}{\operatorname{det}^{\prime} \Delta_{M}}\right)^{1 / 2}(1+Q)^{-\frac{1}{2} \zeta_{\Delta_{M}}(0)-\frac{g}{2}} \\
& \times \sum_{m, n \in \mathbb{Z}^{g}} \exp \left\{-\frac{\pi}{2}\left[\frac{1}{1+Q}(m+n+2 \varepsilon) \operatorname{Im} \Omega_{M}(m+n+2 \varepsilon)\right.\right. \\
& \left.\left.+(1+Q)(m-n) \operatorname{Im} \Omega_{M}(m-n)\right]+2 \pi i(m-n) \kappa\right\},
\end{aligned}
$$

which agrees with formula (23) in [9] up to some numerical factors.

Next, let $M$ be a cylinder, i.e., $g=0, n=1 . \Omega=i \tau_{2}\left(\tau_{2}>0\right)$ is the moduli parameter of the torus and $\widetilde{\Omega}=(1+Q) i \tau_{2}$. The following determinant is well known

$$
\operatorname{det}^{\prime} \Delta_{\Sigma}=\tau_{2}^{2}|\eta(\tau)|^{4}
$$

Thus

$$
Z(Q)=\frac{\vartheta\left[\begin{array}{l}
\delta \\
\lambda
\end{array}\right]\left(0 \mid(1+Q) i \tau_{2}\right)}{\eta\left(i \tau_{2}\right)}
$$


where $\lambda$ describes the spin structure along the closed loop, $\delta=\frac{1}{2}$ or 0 depending on whether the boundary conditions on the two circles are of the same type or not.

We now return to the general case and sum over spin structures when $\delta=0$. The sum over $\kappa$ and $\lambda$ in $2 \pi i(N+E) F=2 \pi i[(m-n) \kappa+l \lambda]$ gives the factor $\prod_{i=1}^{g}\left(1+(-1)^{m_{i}-n_{i}}\right) \prod_{k=1}^{n}\left(1+(-1)^{\lambda_{k}}\right)$, which implies that only $m-n \in(2 \mathbb{Z})^{g}, l \in(2 \mathbb{Z})^{n}$ terms contribute. Let $m^{\prime}=\frac{m-n}{2} \in \mathbb{Z}^{g}, n^{\prime}=m+n+2 \varepsilon \in \mathbb{Z}^{g}$ and $l^{\prime}=\frac{l}{2} \in \mathbb{Z}^{n}$, then

$$
\begin{aligned}
\sum_{\text {spin }} Z(Q)= & \left(\frac{2 \operatorname{det}(\operatorname{Im} \Omega) \operatorname{vol}(M)}{\operatorname{det}^{\prime} \Delta_{\Sigma}}\right)^{1 / 4} 2^{g+n}(1+Q)^{-\frac{1}{2} \zeta_{\Delta_{D}}(0)-\frac{g}{2}} \\
& \times \sum_{m^{\prime}, n^{\prime} \in \mathbb{Z}^{g}, l^{\prime} \in \mathbb{Z}^{n}} \exp \left[-\frac{\pi}{4(1+Q)} N \operatorname{Im} \Omega N\right. \\
& -\pi(1+Q) M \operatorname{Im} \Omega M+i \pi N \operatorname{Re} \Omega M]
\end{aligned}
$$

where

$$
M=\left(\begin{array}{c}
m^{\prime} \\
m^{\prime} \\
2 l^{\prime}
\end{array}\right), \quad N=\left(\begin{array}{c}
n^{\prime} \\
-n^{\prime} \\
0
\end{array}\right) .
$$

Again, if $M$ has no boundaries, the above result agrees with [9].

\section{Bosonization}

In two dimensions, certain fermionic and bosonic theories are equivalent in the sense that there is a (non-local) transformation from fermionic to bosonic field such that interesting physical quantities like the partition function and correlation functions are the same in the two theories. This idea is called bosonization and was first discovered in the massive Thirring model [24]. Bosonization of first order fermionic system on a Riemann surface of arbitrary genus has been studied in detail $[2,7,8]$. In this section, we start with a bosonized theory on a general Riemann surface with boundaries and show that its partition coincides with that of the massless Thirring model of fermion computed in Sect. 4.

We replace the fermionic determinant in (4.1) by [12]

$$
\int \mathscr{D} \phi \mathrm{e}^{-\frac{1}{2 \pi}\langle d \phi+A, d \phi+A\rangle_{M}}=\int \mathscr{D} \phi \mathrm{e}^{-\frac{1}{2 \pi} \int_{M} d^{2} \sigma \sqrt{g} g^{\alpha \beta}\left(\partial_{\alpha} \phi+A_{\alpha}\right)\left(\partial_{\beta} \phi+A_{\beta}\right)},
$$

where $\phi: M \rightarrow S^{1}$ is a circle valued scalar field on the Riemann surface $M$. As in the fermionic theory, $\phi$ has to satisfy the Neumann boundary condition $\left.\frac{\partial \phi}{\partial n}\right|_{\partial M}=0$ such that the boundary terms from the variation of the action vanish [18]. The action is invariant under local gauge transformations $A \rightarrow A^{\prime}=A+d s, \phi \rightarrow \phi^{\prime}$ 
$=\phi-s$. If the boundary condition on $\phi$ is preserved, the Neumann boundary condition on $s$ implies once more that the vector potential $A$ can be chosen to satisfy the absolute boundary condition.

The partition function is

$$
\begin{aligned}
Z(Q) & =\frac{\int \mathscr{D} A \mathrm{e}^{-\frac{1}{2 \pi Q}\langle A, A\rangle_{M}} \int \mathscr{D} \phi \mathrm{e}^{-\frac{1}{2 \pi}\langle d \phi+A, d \phi+A\rangle_{M}}}{\int \mathscr{D} A \mathrm{e}^{-\frac{1}{2 \pi \mathscr{Q}}\langle A, A\rangle_{M}}} \\
& =\frac{\int \mathscr{D} \phi \mathrm{e}^{-\frac{1}{2 \pi(1+Q)}\langle d \phi, d \phi\rangle_{M}} \int \mathscr{D} A \mathrm{e}^{-\frac{1}{2 \pi}\left(\frac{1}{Q}+1\right)\left\langle A+\frac{1}{\frac{1}{Q}+1} d \phi, A+\frac{1}{\frac{1}{Q}+1} d \phi\right\rangle_{M}}}{\int \mathscr{D} A \mathrm{e}^{-\frac{1}{2 \pi Q}\langle A, A\rangle_{M}}} .
\end{aligned}
$$

After a Gaussian shift $A \rightarrow A+\frac{1}{1} d \phi$ in the numerator, the integral over $A$ is $\frac{1}{Q}+1$

simple,

$$
\begin{aligned}
Z(Q)= & \left(\frac{\operatorname{det}^{\prime} \frac{1}{Q} \Delta_{N}}{\operatorname{det}^{\prime}\left(\frac{1}{Q}+1\right) \Delta_{N}}\right)^{1 / 2}\left(\frac{\operatorname{det}^{\prime} \frac{1}{Q} \Delta_{D}}{\operatorname{det}^{\prime}\left(\frac{1}{Q}+1\right) \Delta_{D}}\right)^{1 / 2}\left(\frac{\frac{1}{Q}}{\frac{1}{Q}+1}\right)^{1 / 2} \\
& \times \int \mathscr{D} \phi \mathrm{e}^{-\frac{1}{2 \pi(1+Q)}\langle d \phi, d \phi\rangle_{M}} \\
= & (1+Q)^{-\frac{1}{2} \zeta_{\Delta_{N}}(0)-\frac{1}{2} \zeta_{\Delta_{D}}(0)-g-\frac{n}{2}} \int \mathscr{D} \phi \mathrm{e}^{-\frac{1}{2 \pi(1+Q)}}\langle d \phi, d \phi\rangle_{M}
\end{aligned}
$$

Effectively, this is equivalent to the theory of a free scalar field which takes its value on a circle of radius shifted by a factor of $(1+Q)^{-1 / 2}[12]$.

The precise meaning of being $S^{1}$-valued is the decomposition of $\phi$ into the fluctuation and the soliton part [2], i.e.,

$$
d \phi=d \phi^{\prime}+\sum_{m, n \in\left(\frac{1}{2} \mathbb{Z}\right)^{g}, l \in\left(\frac{1}{2} \mathbb{Z}\right)^{n}}\left(\alpha-\alpha^{\prime}\right) \cdot n-\left(\beta+\beta^{\prime}\right) \cdot m-\delta \cdot l,
$$

where the $\mathbb{R}$-valued function $\phi^{\prime} \in \Omega_{N}^{0}(M)$ is the nonzero mode of the fluctuation and the sum is the soliton part. Therefore,

$$
\begin{aligned}
& \int \mathscr{D} \phi \mathrm{e}^{-\frac{1}{2 \pi Q}\langle d \phi, d \phi\rangle_{M}}=(\operatorname{vol}(M))^{1 / 2}\left(\operatorname{det}^{\prime} \frac{1}{1+Q} \Delta_{N}\right)^{-1 / 2} \\
& \times \sum_{m, n \in \mathbb{Z}^{g}, l \in \mathbb{Z}^{n}} \exp \left[-\frac{\pi}{4(1+Q)}\left(N \bar{\Omega}+M^{\prime}\right)(\operatorname{Im} \Omega)^{-1}\left(\Omega N+M^{\prime}\right)\right],
\end{aligned}
$$

where

$$
N=\left(\begin{array}{c}
n \\
-n \\
0
\end{array}\right), \quad M^{\prime}=\left(\begin{array}{c}
m \\
m \\
l
\end{array}\right)
$$


The factor $(\operatorname{vol}(M))^{1 / 2}$ is the contribution of the zero mode. The determinant of the Laplacian with Neumann boundary condition is $[4,5]$

$$
\operatorname{det}^{\prime} \Delta_{N}=\left(2 \operatorname{vol}(M) \operatorname{det}^{\prime} \Delta_{\Sigma}\right)^{1 / 2}\left(\frac{\operatorname{det}^{\prime} P_{+} \operatorname{Im} \Omega P_{+}}{\operatorname{det}^{\prime} P_{-} \operatorname{Im} \Omega P_{-}}\right)^{-1 / 2} .
$$

The sum over $m$ and $l$ can be rewritten using the Poisson summation formula (see, e.g. [25]),

$$
\begin{aligned}
\sum_{m \in \mathbb{Z}^{g}, l \in \mathbb{Z}^{n}} \exp \left[-\frac{\pi}{4(1+Q)} M^{\prime}(\operatorname{Im} \Omega)^{-1} M^{\prime}-2 M^{\prime}(\operatorname{Im} \Omega)^{-1} \operatorname{Re} \Omega N\right] \\
=\frac{\pi^{\frac{g+n}{2}}}{\left(2^{g} \operatorname{det}^{\prime} P-\frac{\pi}{4(1+Q)}(\operatorname{Im} \Omega)^{-1} P_{-}\right)^{1 / 2}} \exp \left[N \operatorname{Re} \Omega(\operatorname{Im} \Omega)^{-1} \operatorname{Re} \Omega N\right] \\
\quad \times \sum_{m \in \mathbb{Z}^{g}, l \in \mathbb{Z}^{n}} \exp [-\pi(1+Q) M \operatorname{Im} \Omega M+i \pi N \operatorname{Re} \Omega M],
\end{aligned}
$$

where

$$
M=\left(\begin{array}{c}
m \\
m \\
2 l
\end{array}\right)
$$

So the partition function of the bosonized theory is

$$
\begin{aligned}
Z(Q)= & \left(\frac{\operatorname{det}(\operatorname{Im} \Omega) \operatorname{vol}(M)}{2 \operatorname{det}^{\prime} \Delta_{\Sigma}}\right)^{1 / 4} 2^{\frac{g}{2}+n}(1+Q)^{-\frac{1}{2} \zeta_{\Delta_{D}}(0)-\frac{g}{2}} \\
& \times \sum_{m, n \in \mathbb{Z}^{g}, l \in \mathbb{Z}^{n}} \exp \left[-\frac{\pi}{4(1+Q)} N \operatorname{Im} \Omega N\right. \\
& -\pi(1+Q) M \operatorname{Im} \Omega M+i \pi N \operatorname{Re} \Omega M] .
\end{aligned}
$$

This agrees with the fermionic calculation (4.19) up to factors which depend on neither the moduli parameters nor the coupling constant.

\section{Appendix. Calculation of the Zeta-functions at Zero}

We compute the values of $\zeta_{\Delta_{N}}(0)$ and $\zeta_{\Delta_{D}}(0)$ which are used in Sects. 4 and 5.

Recall that for a non-negative self-adjoint operator $A$ acting on a Hilbert space, the zeta-function of $A$ is defined by $[11,26]$

$$
\zeta_{A}(s)=\operatorname{tr}^{\prime} A^{-s}=\sum^{\prime} \frac{1}{\lambda_{n}^{s}},
$$

where prime means summing over the nonzero eigenvalues $\lambda_{n} \neq 0$ only. The zetafunction regularization of the determinant [23]

$$
\operatorname{det}^{\prime} A=\mathrm{e}^{-\zeta_{A}^{\prime}(0)}
$$


implies property (4.7). If $A$ is an elliptic pseudodifferential operator of degree $m$ on a compact manifold of dimension $d$, the above sum is absolutely convergent when $\operatorname{Re} s>\frac{d}{m}$, hence $\zeta_{A}(s)$ is holomorphic in that region. It was shown [27] that the $\zeta_{A}(s)$ can be extended analytically to a meromorphic function on the whole complex plane, with poles which can be situated only at the points $s_{j}=-\frac{j-d}{m}, j=0,1, \ldots$, or at the negative integers. In particular, $\zeta_{A}(0)$ is finite.

The zeta-function can be computed using the heat kernel. In fact,

$$
\zeta_{A}(s)=\frac{1}{\Gamma(s)} \int_{0}^{\infty} t^{s-1} \operatorname{tr}^{\prime} \mathrm{e}^{-t A} d t
$$

i.e., $\zeta_{A}(s) \Gamma(s)$ is the Mellin transform of the heat kernel $\operatorname{tr}^{\prime} \mathrm{e}^{-t A}[11,26,23]$. The inverse Mellin transform is

$$
\begin{aligned}
\operatorname{tr}^{\prime} \mathrm{e}^{-t A} & =\frac{1}{2 \pi i} \int_{\operatorname{Re} s=c} t^{-s \zeta_{A}}(s) \Gamma(s) d s \\
& =\sum_{\operatorname{Re} p<c} \operatorname{Res}\left\{t^{-s} \zeta_{A}(s) \Gamma(s) ; p\right\} \\
& =\zeta_{A}(0)+\sum_{p \neq 0} t^{-\operatorname{Re} p} \operatorname{Res}\left\{\zeta_{A}(s) \Gamma(s) ; p\right\} .
\end{aligned}
$$

The above formula makes sense for sufficiently large $c>0$ because of the distribution of the poles of $\zeta_{A}(s)$ and $\Gamma(s)$.

If we know the heat kernel expansion of the operator $A$,

$$
\operatorname{tr}^{\prime} \mathrm{e}^{-t A} \sim \sum_{n} a_{n} t^{n}, \quad \text { as } \quad t \sim 0^{+},
$$

then

$$
\zeta_{A}(0)=a_{0} .
$$

Therefore $\zeta_{A}(0)$ is a topological invariant (see, e.g. [28]). Notice that although $\zeta_{A}(s)$ depends on the scaling of the manifold, its value at $s=0$ is not, just like the $\eta$-invariant in the index theory on manifolds with boundaries $[13,28]$. For the Laplacian on a compact Riemannian manifold $\Sigma$ of dimension $d$, the heat kernel expansion is $[29,30,28]$

$$
\operatorname{tre}^{-t \Delta_{\Sigma}}=\frac{1}{(4 \pi t)^{d / 2}}\left[\operatorname{vol}(\Sigma)+\frac{t}{6} \int_{\Sigma} R+o\left(t^{2}\right)\right], \text { as } t \sim 0^{+},
$$

where $R$ is the Ricci curvature scalar. If $\Sigma$ is a Riemann surface of genus $h$, then applying the Gauss-Bonnet theorem,

$$
\operatorname{tre}^{-t \Delta_{\Sigma}}=\frac{\operatorname{vol}(\Sigma)}{4 \pi t}-\frac{1}{3}(h-1)+o(t), \text { as } t \sim 0^{+} .
$$

Hence

$$
\zeta_{\Delta_{\Sigma}}(0)=-\frac{1}{3}(h-1)-1
$$


Let $M$ be a Riemann surface of genus $g$ with $n+1$ components of boundaries. $\Delta_{N}$ and $\Delta_{D}$ are the Laplacians acting on the functions with Neumann and Dirichlet boundary conditions on $M$. These spaces $\Omega_{N}^{0}(M)$ and $\Omega_{D}^{0}(M)$ can be identified respectively with the spaces of functions on the doubled surface $\Sigma$ which are even and odd under the parity $\sigma^{*}$. Hence

$$
\begin{aligned}
\zeta_{\Delta_{N}}(0)+\zeta_{\Delta_{D}}(0) & =\text { coefficient of } t^{0} \text { in } \operatorname{tr}^{\prime} \mathrm{e}^{-t \Delta_{\Sigma}} \\
& =-\frac{1}{3}(2 g+n-1)-1
\end{aligned}
$$

and

$$
\zeta_{\Delta_{N}}(0)-\zeta_{\Delta_{D}}(0)=\text { coefficient of } t^{0} \text { in } \operatorname{tr}^{\prime} \sigma^{*} \mathrm{e}^{-t \Delta_{\Sigma}}
$$

Now

$$
\begin{aligned}
\operatorname{tr} \sigma^{*} \mathrm{e}^{-t \Delta_{\Sigma}} & =\int_{z \in \Sigma} d^{2} z\left\langle z\left|\sigma^{*} \int_{w \in \Sigma} d w\right| w\right\rangle\left\langle w\left|\mathrm{e}^{-t \Delta_{\Sigma}}\right| z\right\rangle \\
& =\int_{z, w \in \Sigma} d^{2} z d^{2} w \delta^{(2)}(z-\sigma w)\left\langle w\left|\mathrm{e}^{-t \Delta_{\Sigma}}\right| z\right\rangle \\
& =\int_{z \in \Sigma} d^{2} z\left\langle z\left|\mathrm{e}^{-t \Delta_{\Sigma}}\right| \sigma z\right\rangle \\
& =\int_{z \in \partial M \times(-\varepsilon, \varepsilon)} d^{2} z\left\langle z\left|\mathrm{e}^{-t \Delta_{\Sigma}}\right| \sigma z\right\rangle+o(t) .
\end{aligned}
$$

In the last step, the integral over $\Sigma$ is changed to one on a tubular neighborhood of $\partial M$ because only pairs of points close to each other have significant contribution to the heat kernel at small $t$. For the same reason, the integral can be rewritten as one on $\partial M \times \mathbb{R}$.

$$
\begin{aligned}
\operatorname{tr} \sigma^{*} \mathrm{e}^{-t \Delta_{\Sigma}} & =\int_{z \in \partial M \times \mathbb{R}} d^{2} z\left\langle z\left|\mathrm{e}^{-t \Delta_{\partial M} \times \mathbb{R}}\right| \sigma z\right\rangle+o(t) \\
& =\int_{x \in \partial M, y \in \mathbb{R}} d x d y\left\langle x\left|\mathrm{e}^{-t \Delta_{\partial M}}\right| x\right\rangle\left\langle y\left|\mathrm{e}^{-t \Delta_{\mathbb{R}}}\right|-y\right\rangle+o(t) \\
& =\int_{x \in \partial M} d x\left\langle x\left|\mathrm{e}^{-t \Delta_{\partial M}}\right| x\right\rangle \int_{y \in \mathbb{R}} d y\left\langle 2 y\left|\mathrm{e}^{-t \Delta_{\mathbb{R}}}\right| 0\right\rangle+o(t) \\
& =\frac{1}{2} \operatorname{tr} \mathrm{e}^{-t \Delta_{\partial M}}+o(t) .
\end{aligned}
$$

Therefore,

$$
\begin{aligned}
\zeta_{\Delta_{N}}(0)-\zeta_{\Delta_{D}}(0) & =\frac{1}{2}\left\{\text { coefficient of } t^{0} \text { in } \operatorname{tr} \sigma^{*} \mathrm{e}^{-t \Delta_{\partial M}}\right\}-1 \\
& =\frac{1}{2}(n+1)\left[\zeta_{\Delta_{s^{1}}}(0)+1\right]-1 \\
& =\frac{1}{2}(n+1)[2 \zeta(0)+1]-1 \\
& =-1
\end{aligned}
$$

Here we have used

$$
\zeta_{\Delta_{s^{1}}}(s)=2 \sum_{n=1}^{\infty} \frac{1}{\left(n^{2}\right)^{s}}=2 \zeta(2 s)
$$


where $\zeta(s)$ is the Riemann zeta-function. Finally, from (A.10) and (A.14), we get

$$
\begin{gathered}
\zeta_{\Delta_{N}}(0)=-\frac{1}{6}(2 g+n-1)-1, \\
\zeta_{\Delta_{D}}(0)=-\frac{1}{6}(2 g+n-1) .
\end{gathered}
$$

Acknowledgements. I am grateful to D. Z. Freedman for proposing this problem, for much help and encouragement throughout the course of this work and for reading the manuscript. I would like to thank J. Edward, R. Melrose, P. Piazza, K. Pilch, and C. Vafa for stimulating discussions. Finally, I thank E. Tomboulis for bringing to my attention Ref. [22]. This work was supported in part by a Wang fellowship.

\section{References}

1. D'Hoker, E., Phong, D.: On determinants of Laplacians on Riemann surfaces. Commun. Math. Phys. 104, 537 (1986)

2. Alvarez-Gaumé, L., Moore, G., Vafa, C.: Theta functions, modular invariance, and strings. Commun. Math. Phys. 106, 1 (1986)

3. Alvarez, O.: Theory of strings with boundary. Nucl. Phys. B 216, 125 (1983)

4. Blau, S., Clements, M., Della Pietra, S., Carlip, S., Della Pietra, V.: The string amplitude on surface with boundaries and crosscaps. Nucl. Phys. B 301, 285 (1988)

5. Morozov, A., Rosly, A.: On many-loop calculations in the theory of open strings. Phys. Lett. B 214, 522 (1988)

6. Quillen, D.: Determinants of Cauchy-Riemann operators on Riemann surfaces. Funkts. Anal. Prilozh. 19, 37 (1985)

7. Alvarez-Gaumé, L., Bost, J.B., Moore, G., Nelson, P., Vafa, C.: Bosonization in arbitrary genus. Phys. Lett. B 178, 41 (1986); Bosonization on higher genus Riemann surfaces. Commun. Math. Phys. 112, 503 (1987)

8. Verlinde, E., Verlinde, H.: Chiral bosonization, determinants and the string partition function. Nucl. Phys. B 288, 357 (1987)

9. Freedman, D., Pilch, K.: Thirring model partition functions and harmonic forms. Phys. Lett. B 213, 331 (1988)

10. Conner, P.: The Neumann's problem for differential forms on Riemannian manifolds. Mem. Am. Math. Soc. 20, (1956)

11. Ray, D., Singer, I.: $R$-torsion and the Laplacian on Riemannian manifolds. Adv. Math. 7, 145 (1971)

12. Freedman, D., Pilch, K.: Thirring model on a Riemann surface. MIT preprint CTP \# 1701 (January 1989). Ann Phys. (to appear)

13. Atiyah, M., Patoldi, V., Singer, I.: Spectral asymmetry and Riemannian geometry. I. Math. Proc. Camb. Phil. Soc. 77, 43 (1975)

14. Fay, J.: Theta functions on Riemann surfaces. Lecture Notes in Mathematics vol. 352. Berlin, Heidelberg, New York: Springer 1973

15. Mumford, D.: Theta characteristics of an algebraic curve. Ann. Sci. Éc. Norm. Sup. 4, 181 (1971)

16. Mumford, D.: Curves and their Jacobians. Ann Arbor: Michigan 1976

17. Mumford, D.: Tata lectures on theta, Vol. I. Boston: Birkhäuser 1983

18. Green, M., Schwarz, J., Witten, E.: Superstring theory. Cambridge: Cambridge University Press 1987

19. Blau, S., Visser, M., Wipf, A.: Determinants, Dirac operators, and one-loop physics. Int. J. Mod. Phys. A 4, 1467 (1989)

20. Witten, E.: An SU(2) anomaly. Phys. Lett. B 117, 324 (1982)

21. Igusa, J.: Theta functions. Berlin, Heidelberg, New York: Springer 1972

22. Tomboulis, E.: Gauge fields on the world sheet: Strings with gauge- and supersymmetric breaking. Phys. Lett. B 198, 165 (1987) 
23. Hawking, S.: Zeta function regularization of path integral in curved spacetime. Commun. Math. Phys. 55, 133 (1977)

24. Coleman, S.: Quantum sine-Gordon equation as the massive Thirring model. Phys. Rev. D 11, $2088(1975)$

25. Serre, J.-P.: A course in arithmetic. Berlin, Heidelberg, New York: Springer 1973

26. Ray, D., Singer, I.: Analytic torsion for complex manifolds. Ann. Math. 98, 154 (1973)

27. Shubin, M.: Pseudodifferential operator and spectral theory. Berlin, Heidelberg, New York: Springer 1987

28. Gilkey, P.: Invariance theory, the heat equation, and the Atiyah-Singer index theorem. Wilmington, Publish or Perish 1984

29. McKean, H., Singer, I.: Curvature and the eigenvalues of the Laplacian. J. Diff. Geom. 1, 43 (1967)

30. Chavel, I.: Eigenvalues in Riemannian geometry. Orlando: Academic Press 1984

Communicated by A. Jaffe

Received January 5, 1989; in revised form March 2, 1989 